\title{
ACERCAMIENTOS Y ACUERDOS COMERCIALES ENTRE GRANADA Y VENECIA AL FILO DE $1400^{1}$
}

\author{
COMMERCIAL AGREEMENTS BETWEEN \\ NASRID KINGDOM OF GRANADA AND VENICE AT 1400
}

\section{ADELA FÁBREGAS GARCÍA Universidad de Granada}

\begin{abstract}
Resumen: Los preparativos dispuestos en 1400 por el Senado veneciano para el establecimiento de acuerdos comerciales con el reino nazarí de Granada, que conocemos a través de documentación de los archivos venecianos, constituyen un ejemplo de la utilidad de la vía diplomática como recurso de desarrollo económico. Permiten, además, reconocer la actitud de apertura de las autoridades nazaríes frente a la dinamización comercial de sus territorios, facilitando al máximo nuevas incorporaciones de las principales comunidades de negocios del Occidente bajomedieval.
\end{abstract}

Palabras clave: Diplomacia; Comercio; Venecia; Reino Nazarí; Comunidades mercantiles.

\begin{abstract}
An example of the fruitfulness of diplomacy as a resource for economic development may be found in the arrangements disposed in 1400 by the Venetian Senate to establish commercial agreements with the Nasrid kingdom of Granada. Those agreements show also an open attitude from the Nasrid authorities facing a commercial stimulation of their territories, easing greatly new incorporations of the leading business communities from the Late Medieval Western world.
\end{abstract}

Keywords: Diplomacy; Commerce; Venice; Nasrid Kingdom; Merchant communities.

\section{SUMARIO}

1. Introducción.- 2. Venecia abre un nuevo espacio de interés.- 3. La negociación diplomática.4. Los intereses a tutelar.- 5. Epílogo.- 6. Apéndice documental.

\section{INTRODUCCIÓN}

Si hay un ejemplo cumplido de dependencia estrecha de un estado respecto al juego diplomático, éste podría ser sin duda el del reino nazarí de Granada. Su supervivencia se relaciona, por momentos de manera crucial, precisamente con la habilidad diplomática de sus gobernantes, con el

${ }^{1}$ El presente estudio ha sido realizado gracias a una ayuda concedida por la Junta de Andalucía, Consejería de Innovación, Ciencia y Empresa (Programa de Estancias de Excelencia 2008/I), para la realización de trabajos de investigación en la Università Ca' Foscari di Venezia. 
impecable uso que hacen de la misma y con la optimización máxima de los distintos desarrollos que esta opción política puede tener.

Así, de todos es sabido que el nacimiento mismo de este pequeño estado "oportunista", surgido a partir de la descomposición del gran imperio almohade y lastrado por fuertes dudas acerca de su legitimidad ${ }^{2}$ y soberanía, es protegido por la confección de acuerdos, que resuelven o al menos marcan la senda por la que debe dirigirse la existencia política del reino, una realidad con limitaciones, pero vigente al fin y al cabo.

Está, por tanto, en la naturaleza misma de este estado la actitud que mostrará de manera recurrente a lo largo de toda su historia, hasta el punto de que puede considerarse como una de las características propias de la dinámica política nazarí: el continuo recurso a acuerdos y pactos con las potencias territoriales vecinas y con las fuerzas interiores representadas por la nobleza. Esta política de equilibrios, de acuerdos, de juego diplomático muy sutil y complicado, va a marcar la política internacional del reino y la historia nazarí en general. Los protagonistas de la misma van a ser las grandes potencias mediterráneas, en particular la Corona de Aragón y Génova, los estados del Magreb, fundamentalmente el Estado merini de Fes (benimerines), y Castilla, por supuesto. Es en este campo donde busca, donde encuentra, donde se labra su supervivencia, preservando su integridad territorial.

Pero también, cómo no, es el espacio donde es capaz de afianzar una estabilidad económica que es condición indispensable, tan importante o más, a la hora de sellar una garantía de perdurabilidad. Y es que ambas exigencias, la de la estabilidad político-territorial y la de una estabilidad económica, se ven suficientemente cubiertas trabajando bajo el amparo de la vía diplomática, que desarrolla en estos momentos dos líneas de actuación muy bien definidas en sendas tradiciones, bajo el sistema de treguas o tratados de paz (vinculado a relaciones de guerra) o de acuerdos comerciales. Nos explicamos rápidamente.

Sin entrar ni mucho menos en el tema, sí que hay que recordar que cada vez con mayor rotundidad se habla de una estrategia de fortalecimiento comercial abordada desde los más altos estamentos de poder nazarí. Se trata de apuntalar económicamente esta dinastía reinante. Y se trata también de asegurar un estado próspero. Nada mejor que acudir a la vía comercial como espacio de prosperidad, aprovechando el impulso económico construido a partir del naciente sistema de intercambios que cubre todo el Occidente bajomedieval. No es, pues, sorprendente la dinámica en la que se embarca

${ }^{2}$ Lo que constituye una preocupación constante y marca la actuación de buena parte de los soberanos nazaríes en su busqueda constante de instrumentos capaces de revestir su poder de autoridad. Véanse, por ejemplo, recientes tratamientos del tema en Emilio MOLINA LOPEZ, La dinámica política y los fundamentos del poder, en Historia del Reino de Granada. I. De los orígenes a la época mudéjar (hasta 1502) (R. PEINADO SANTAELLLA, ed.), Granada 2000, pp. 211-248; Virgilio MARTINEZ ENAMORADO, "Lema de príncipes". Sobre la Galiba y algunas evidencias epigráficas de su uso fuera del ámbito nazarí, "Al-Qantara” XXVII/2 (2006), pp. 529 550; María Jesús RUBIERA MATA, El califato nazari, "Al-Qantara” XXIX/2 (2008), pp. 293-305; Antonio PELÁEZ ROVIRA, El emirato nazarí de Granada en el siglo XV. Dinámica política y fundamentos sociales de un estado andalusí, Granada, 2009, pp. 59-121. 
este pequeño reino del Mediterráneo, que una vez abre y explora esta vía de desarrollo, una vez que la misma demuestra sus enormes posibilidades, su potencial como tabla de salvación, no deja de auspiciar su progresión ${ }^{3}$.

Todo ello deviene en una progresiva apertura por parte de las autoridades nazaríes a un espectro cada vez más amplio y diversificado de comunidades mercantiles, mostrando una notable flexibilidad a la hora de acoger nuevas iniciativas. Una política no restrictiva ${ }^{4}$ que favorece, o puede favorecer, un juego de competencia absolutamente beneficioso para los intereses nazaríes.

Evidentemente este impulso, esta protección que se brinda al comercio internacional y a sus protagonistas tiene en la baza diplomática uno de sus instrumentos más eficaces. De hecho se muestran extraordinariamente atentos a la apertura de relaciones diplomáticas y comerciales con las grandes potencias económicas del momento, con Génova, sobre todo, pero también la Corona de Aragón o Venecia, a inicios del siglo XV, en pleno momento de esplendor comercial nazarí.

En este campo aprovechan la extraordinaria experiencia desarrollada por las potencias comerciales italianas, interlocutores comerciales de primer orden para el reino nazarí, y que en palabras de Petti Balbi, han convertido la iniciativa político-diplomática y la actividad mercantil en los dos pilares básicos sobre los que sustentan desde el siglo XII todo su sistema de relaciones con el mundo islámico ${ }^{5}$. Porque, aunque las diferentes comunidades mercantiles que van floreciendo progresivamente a lo largo de los principales

\footnotetext{
${ }^{3}$ Una opción asumida por los reyes nazaríes que se manifiesta a diversos niveles. Desde los apoyos oficiales que auspician la creación de infraestructuras necesarias o la puesta en marcha de una política fiscal favorable en materia de comercio, hasta una participación directa de la casa real en actividades de este tipo, como proveedores de bienes de comercio e incluso como protagonistas activos en la red de distribución internacional de productos nazaríes. José Enrique LOPEZ DE CoCA, Granada y la ruta de poniente: el tráfico de frutos secos (siglos XIV-XV), en Navegación marítima del Mediterráneo al Atlántico (Antonio MALPICA CUELLO, ed.), Granada, 2001, pp. 149-177; Antonio MALPICA CUELLO, Entre la arqueología y la historia. Castillos y poblamiento en Granada. Estudio de una política edilicia a partir de la Alhambra, en XXII Semana de Estudios Medievales. Estella 1995. Tecnología y Sociedad: Las grandes obras públicas en la Europa Medieval, Pamplona, 1996, pp. 289-326; IDEM, La expansión urbana de la Granada nazarí y la acción de los reyes granadinos, en Homenaje al profesor Angel Barrios García, Salamanca, 2007, pp. 133-153; Roser SALICRÚ, Génova y Castilla, genoveses y Granada. Política y comercio en el Mediterráneo occidental en la primera mitad del siglo XV (1431-1439), en Le vie del Mediterraneo. Idee, uomini, oggetti (secoli XI-XVI), (Gabriella AIRALDI G. a cura di), Génova, 1997, pp. 213-257; Adela FABREGAS GARCÍA, Actividad comercial de los reyes nazaríes y su implicaciọn con los representantes del gran comercio occidental a finales de la Edad Media. "Studia Historica", 25 (2007), pp. 171-190

${ }^{4}$ Observable en la enorme tonicidad de los negocios, intereses y colaboraciones que aún mantiene la comunidad genovesa en el reino a los más altos niveles. José Enrique LóPEZ DE CocA, Génova y el Reino de Granada (siglos XIII-XV), en Relazioni economiche tra Europa e mondo islámico secc. XIII-XVIII. Atti della Trentottesima Settimana di Studi, 1-5 maggio 2006 (S. CAVACIOCCHI a cura di), Firenze, 2007, pp. 267-294; Roser SALICRÚ, ¿Repensando Granada? Presencia y penetración diferencial cristiana en el sultanato nazarí en la Baja Edad Media, en Relazioni economiche tra Europa e mondo islámico, pp. 135-146.

${ }^{5}$ Giovanna PETTI BALBI, Las ciudades marítimas italianas y el norte de Africa en época medieval: relaciones políticas y económicas, en Relaciones entre el Mediterráneo cristiano y el norte de Africa en época medieval y moderna (C. TRILLO ed), Granada 2004 pp. 19-51, espec. p. 19. Antonio MALPICA CUELLO, Dal Mediterraneo islamico al Mediterraneo cristiano. II dominio del bacino occidentale nel Mediterraneo centrale, en Una città nel Mediterraneo. l'Opulenta Salernum (V. D'ARIENZO, ed.), Salerno, 2001, pp. 31-66
} 
mercados y riberas del Mediterráneo y del Atlántico sean fruto de la voluntad privada, en todos los casos, lo cierto es que de ningún modo verán garantizada su continuidad y prosperidad si no es con el apoyo externo, con la adhesión a su programa económico de la voluntad política de los estados de procedencia y de acogida de esos grupos mercantiles, de manera que se garanticen y tutelen esos intereses privados. De ahí que un hecho más o menos espontáneo, como es la apertura de nuevos espacios de interés comercial, devenga más temprano que tarde en un programa institucional estable, provisto de privilegios, estatutos y claves de tratamiento pormenorizado que permitan ordenar esas actuaciones privadas. A definir este tipo de instituciones se dedica la actividad diplomática de estas grandes potencias comerciales, que generan un tipo específico de acuerdos basados de manera exclusiva en la tutela comercial de sus intereses ${ }^{6}$.

Así que hay una diplomacia que no trabaja al servicio de la paz, o de la guerra. Existe una diplomacia que trabaja, que siempre ha trabajado al servicio del dinero. El predominio alcanzado por las grandes potencias marítimas mediterráneas no se construye a partir de una hegemonía territorial, sino de un control económico estricto de espacios amplios, desarrollado a partir de la baza comercial. Espacios que, como el granadino, deben preservar su integridad territorial, pero también, cómo no, garantizar su supervivencia económica.

En esa clave acceden muchas de estas grandes potencias comerciales a las tierras nazaríes, y es en este contexto donde adquiere todo su sentido el episodio que tenemos la intención de presentar a continuación y que toca de lleno a una de las comunidades mercantiles más sólidas del Occidente europeo: Venecia.

\section{VENECIA ABRE UN NUEVO ESPACIO DE INTERÉS}

El interés veneciano por las tierras nazaríes queda plenamente integrado en el viraje político-económico adoptado por el gobierno de la Serenissima, que, desde mediados del siglo XIV ha fijado su atención en los puertos y mercados de la Península Ibérica. Con la ampliación hacia Poniente del sistema de mude se refuerza el papel de Venecia como intermediaria entre los dos grandes polos comerciales de Oriente y Occidente. Una apuesta, ésta, formulada ya desde sus primeras etapas por la república véneta como potencia

\footnotetext{
${ }^{6}$ Giovanna PETTI BALBI, Un sistema di negoziazioni politico-commerciali: $i$ consolati genovesi nelbasso medioevo, en Negociar en la Edad Media. Actas del coloquio celebrado en Barcelona en octubre de 2004 (M.T. FERRER MALLOL, J.M. MOEGLIN, S. PÉQUiGnOT, M. SÁNCHEZ MARTíNEZ, eds.), Barcelona, 2005, pp. 476-487.
} 
comercial $^{7}$ y que le está reportando enormes beneficios. El sistema de $m u d e^{8}$, que no se sabe exactamente cuando se adopta ${ }^{9}$, está en la base del impulso comercial de Venecia como potencia económica en Occidente, constituyendo en gran medida el secreto de su prosperidad en un espacio ya consolidado como área de influencia de otras potencias mercantiles, concretamente de Génova. En primer lugar porque la participación decisiva del Estado en este sistema de comercio permite a grandes fortunas, pero también a pequeños y medianos mercaderes, optar por una práctica de comercio internacional a larga distancia, desarrollando negocios, en la medida de sus posibilidades, que de otro modo quedarían totalmente fuera de su alcance ${ }^{10}$. Además, utiliza el medio de transporte más típico de Venecia, la galera, particularmente segura y ágil, lo que facilita el hecho de que los viajes que se organizan se atengan a unas fechas determinadas y a unos movimientos muy precisos, favoreciendo la previsión económica, tanto de inversores de capital como de potenciales clientes, tan necesaria para el éxito de negocios que cuentan con la participación de diversos mercados ${ }^{11}$.

Por último, su disposición a operar en múltiples plazas, y no sólo en Venecia, garantiza un enorme dinamismo y una capacidad de actuar en estos mercados, en condiciones, a veces de monopolio, que reportarán enormes beneficios a los comerciantes venecianos que actúan en calidad de intermediarios. Su interés inicial en este sentido podría ser, tal y como proponen algunos autores, el de controlar eventuales situaciones de desestabilización del mercado veneciano y movimientos especulativos asociados a las mismas ${ }^{12}$. Es así como observamos una evolución desde los momentos iniciales de establecimiento de las mude, a mediados del siglo XIV, con viajes cerrados, hacia una progresiva apertura de escalas que exploran diferentes mercados de las riberas mediterráneas y los abren a la presencia y actividad veneciana.

\footnotetext{
${ }^{7}$ Jean Claude HoCQUET, La organización del comercio veneciano en los siglos XI-XII, en Comercio marítimo en el Mediterraneo medieval y moderno, Granada, 2002, pp. 207-238.

${ }^{8}$ El término muda designa, en palabras de Stöckly, una operación comercial definida que se desarrolla en un plazo de tiempo fijado por el Estado, en un viaje de ida y vuelta con un destino fijo y durante un periodo limitado. La muda es, por tanto, el viaje comercial propiamente dicho, la duración del mismo y las escalas y plazos de tiempo fijados para cargar en las mismas Doris STÖCKLY, Le système de l'Incanto des galées du marché à Venise (fin XIIIe-milieu XVe siècle), Leiden, 1995, p. 26; Alberto TENENTI A Corrado VIVANTI, Le film d'un grand système de navigation: les galères vénitiennes, XIVe-ẊVIe siècles. "Annales", 16/1 (1961), pp. 83-86; Bernard DoUMERC y Doris STÖCKLY, L'évolution du capitalisme marchand a Venise: le financiament des galere da mercato à la fin du XVe siècle. "Annales ESC", 1995 (1), pp. 133157; Jean Claude HoCQUET, Denaro, navi e mercato a Venezia, 1200-1600, Roma, 1999.

${ }^{9}$ Posiblemente a principios del siglo XIV, siendo el año de 1338 el de su instalación permanente. La primera noticia que se tiene acerca de su existencia data del año 1301. Jean Claude HOCQUET, Les routes maritimes du commerce vénitien aux XVe et XVe siècles, en Atti del $V$ Convegno Internazionale di Studi Colombiani. "Navi e navigazione nei secoli XV e XVI", Genova, 1990 vol. II, pp. 581-605.

${ }^{10}$ Doris STÖCKLY, Aspects de la "colonisation vénitienne": Commerce d'État et mobilité sociale au XIV siècle, en Le partage du Monde. Echanges et colonisation dans la Méditerranée médiévale (M. BALARD et A. DUCELLIER eds.), Paris, 1998, pp. 49-61.

${ }^{11}$ J.C. HoCQUET, Denaro, navi e mercato, p. 204 y ss.

${ }^{12}$ ÍDEM, Ibidem.
} 
Precisamente la muda de Flandes, que inicia sus viajes, intermitentes al principio, a partir de 1315 , y que se consolida como línea regular a partir de finales del siglo $\mathrm{XIV}^{13}$, inaugurando los contactos venecianos con los puertos y mercados nazaríes, resulta ser una de las más prosperas en este sentido. Con destinos finales perfectamente definidos, se auspicia una práctica de cabotaje a lo largo de la ruta que, con una selección cuidadosa de las escalas contempladas en la misma, constituye una fuente de beneficios acumulados ${ }^{14}$. Lo cierto es que desde principios del siglo XV empiezan a tomar densidad y regularidad el conjunto de noticias de galeras y escalas venecianas en tierras granadinas y de hecho, la famosa muda de Flandes incluye en sus escalas los puertos nazaríes ${ }^{15}$. Granada se abre como mercado a Venecia, y así se proyecta claramente en los libros de mercancía que tan minuciosamente acaba de repasar Raúl González y donde aparece por primera vez el reflejo del interés comercial veneciano por estas tierras sólo a mediados del siglo $\mathrm{XV}^{16}$.

Se abre con ello todo un nuevo campo de negocios reforzado por un sistema de navegación libre que recorre ya las rutas occidentales del Mediterráneo, y que rápidamente busca una covertura legal, el amparo oficial de la República para consolidar sus posiciones.

\section{LA NEGOCIACIÓN DIPLOMÁTICA}

$\mathrm{Y}$ es que, siguiendo la tradición veneciana ${ }^{17} \mathrm{y}$ en general recogiendo una vez más el modus operandi característico de las principales republicas comerciales italianas, será al abrigo de tratados donde prospere definitivamente la implantación de comunidades mercantiles venecianas. En el caso de la Península Ibérica, empezamos a encontrar consulados venecianos en tierras de la Corona de Aragón, concretamente en Mallorca, funcionando ya en 1358, mientras que Sevilla acoge a la primera comunidad veneciana en Castilla en época de Juan I, aunque con una fecha de inicio de los contactos aún desconocida ${ }^{18}$.

\footnotetext{
${ }^{13} \mathrm{D}$. STÖCKLY, Le système de l'Incanto, pp. 152 y ss.

${ }^{14} \mathrm{~J}$ ean Claude HOCQUET, Itinerarios del comercio marítimo (siglos XV-XVI), en Comercio marítimo en el Mediterráneo medieval, pp. 143-181.

${ }^{15}$ Jose Enrique LÓPEZ DE COCA, Granada y la ruta de poniente: el tráfico de frutos secos (siglos XIV-XV), en Navegación marítima del Mediterráneo al Atlántico (Antonio MALPICA CUELLO, ed.), Granada, 2001, pp. 149-177.

${ }^{16}$ Raúl GONZÁLEZ ARÉVALO, El reino de Granada entre los manuales de mercaderías y los tratados de aritmétiča italianos bajomedievales. "Revista del Centro de Estudios Históricos de Granada y su Reino" 19 (2007), pp. 141-168.

${ }^{17}$ Hay una ingente bibliografía al respecto. Un pequeño trabajo que resume magistralmente las tendencias de desarrollo comercial de Venecia y la política adoptada en apoyo de las mismas en Jean Claude HOCQUET, La organización del comercio veneciano en los siglos XI-XII en Comercio marítimo en el Mediterráneo medieval y moderno, Granada, 2002, pp. 207-238.

${ }^{18}$ Jose Enrique LÓPEZ DE COCA, Las galeras venecianas de Poniente y Berbería desde la perspectiva española. Medievalismo. Boletín de la Sociedad Española de Estudios Medievales, 16 (2006), pp. 113-172.
} 
La apertura de un consulado veneciano en el reino nazarí era ya conocida a partir de breves noticias, recuperadas hace pocos años por José Enrique López de Coca con la intención de llamar la atención sobre esta presencia apenas entrevista a partir, posiblemente, de 1400, primera fecha conocida de contactos entre Venecia y Granada, y confirmada en $1403^{19}$. Esta vía de negocios, casi sólo intuida como oficial y establecida mediante los breves indicios de que disponíamos hasta el momento, se ha visto plenamente confirmada de manera reciente gracias a nuevos hallazgos documentales localizados en los registros de deliberaciones del Senado Veneciano.

En el caso que a nosotros interesa, las deliberaciones acerca de la oportunidad de establecer un consulado en el reino nazarí se encuentran custodiadas en el Archivio di Stato di Venezia, Sección Senato Misti ${ }^{20}$. El seguimiento que se ha realizado hasta el momento ha quedado muy reducido en el tiempo, ya que nuestro rastreo, limitado a esta sección, se ha centrado en la última década del siglo XIV y principios del XV. Son años cruciales para el seguimiento de lo que no se puede definir más que como episodio, teniendo en cuenta que no cristaliza, al menos que sepamos hasta el momento, en fórmulas de asentamiento y colaboración mejor definidas y más sólidas en el tiempo. Y con ello no pretendemos ni mucho menos restarle importancia. Al contrario. Se trata de un episodio extraordinariamente significativo, muy indicativo de cuanto hemos intentado proponer: una tendencia generalizada que nos lleva a hablar desde hace tiempo de una apertura de este pequeño estado musulmán al mundo de los negocios occidental, que no se limita a una actuación concreta de un grupo específico, sino que se abre a cualquier vía o modo de incorporación a las tendencias de desarrollo económico de la Europa bajomedieval a través del comercio.

El 4 de marzo de $1400^{21}$, coincidiendo con la apertura del calendario veneciano y atendiendo a las necesidades que plantea la práctica mercantil veneciana establecida en Occidente a partir de la inauguración de la muda de Flandes $(1338)^{22}$, el Senado encabezado por el Dogo Antonio Veniero aprueba la solicitud a las autoridades nazaríes de apertura de un consulado en Málaga. Un consulado que atienda a las necesidades de los mercaderes venecianos, que los acoja en una alhóndiga propia durante sus estancias y tratos en la ciudad ${ }^{23}$, negocios favorecidos por la escala de las galeras en el viaje de Flandes. Se

\footnotetext{
${ }^{19}$ Jose Enrique LÓPEZ DE COCA, Sobre las galeras venecianas de Poniente y sus escalas ibéricas (siglo XV), en Homenaje a Tomás Quesada, Granada, 1998, pp. 401-416

${ }^{20}$ Las deliberaciones del Senado fueron tratadas y reflejadas de forma conjunta hasta el año de 1400 . A partir de 1401, las deliberaciones relativas a materia política quedarán registradas en una serie particular denominada Secreta, mientras que el resto , posteriormente, sólo mucho más adelante, desde 1440 , dividiría su naturaleza y registro en Delibere de Tierra y de Mar. Andrea DA MosTo, L'Archivio di Stato di Venezia. Indice generale, storico, descrittivo ed analitico, I, Roma, 1937.

${ }^{21}$ Archivio di Stato di Venezia, Senato Misti, Reg. XLV, fol. 1 r.

${ }^{22}$ Alberto TENENTI, A., Corrado VIVANTI, Le film d'un grand système; D. STÖCKLY, Le système de l'Incanto des galées, Leiden, 1995.

${ }^{23}$ En realidad consulado y alhóndiga, funduk, resultan ser manifestaciones de la misma realidad diplomática, quedando el funduk, el fóndaco de la documentación italiana, como referencia topográfica, base territorial del poder consular.
} 
acuerda la elección de un embajador y de un cónsul, al que se le asigna un estipendio anual de 400 ducados y tres criados (famuli), uno de los cuales ejercerá de traductor (trujamán), dos caballos y un clérigo, mantenido con un sueldo de 25 ducados que corre a cargo del Estado de la Serenísima.

La elección de los representantes de la misión recae en principio en dos nobles venecianos, Ser Francisco de Bernardo, embajador encargado de contactar con las autoridades granadinas y de negociar este establecimiento, y Ser Bernardo Contarino, cónsul que ostentaría la carga durante los primeros dos años en caso de que las conversaciones entre los dos estados llegaran a buen fin. Los gastos inherentes a toda la operación y a los primeros años de administración consular serán cubiertos a partir de la creación de un derecho que gravará con el $0,5 \%$ de su valor las mercancías que se descarguen en el puerto nazarí, tanto en los viajes oficiales de la Muda de Flandes como mediante cualquier otro tipo de navegación libre ${ }^{24}$. El mismo cónsul quedará encargado de su percepción, garantizando el Senado un eventual apoyo económico hasta completar el montante final de gastos en caso de que estos no sean cubiertos en su totalidad.

Pocos días después, el 23 de marzo ${ }^{25}$, se confirma la iniciativa, confiando el Dogo a Bernardo Contarino la dirección de la expedición como embajador y cónsul en Málaga. Se le explican de manera detallada las líneas de desarrollo que deberá seguir en esta misión, tanto sobre los términos del acuerdo que se podría estipular con las autoridades nazaríes, con el rey granadino, sobre todo en materia fiscal, como respecto a cuestiones meramente organizativas en relación a los servicios y naturaleza del asentamiento veneciano. Se redacta para ello una extensa comisión que consta de 14 puntos en los que se desarrollan las siguientes exigencias:

1. Planteará la creación de una alhóndiga en Málaga, sede del consulado, que acoja a mercaderes venecianos y a sus mercancías de manera cómoda y segura, con la aprobación expresa del rey (debeat dectum fonticum teneri in concio exponsis regiis).

2. Se solicitará a los nazaríes el mantenimiento conjunto de este consulado, tal y como ocurre en los demás asentamientos venecianos en el mundo islámico. Para ello se pedirá una aportación de 200 doblas, que podrán ser rebajadas en la tratativa hasta un mínimo de 100 doblas. En todo caso el interés en estos momentos es tal por parte del gobierno veneciano, que aclaran al cónsul la intención firme de mantener abierto el consulado aún cuando no se consiga ningún apoyo pecuniario por parte de las autoridades nazaríes.

\footnotetext{
24"Et quia non est iustum necque convenens quo comune nostro habeat expensam predicti consulis, ordinetur quo nostri extraordinarii exigere debeant de omnibus mercationibus quos cum galeys et aliis navigiis nostris conducentur de Venetiis ad dectum locum Maliche dimidiam pro centinario quam ponere debeant in nostrum comune et quia de Flandra et aliis locis conducentur ad dectum locum Maliche multe mercationes cum galeys nostris, ordinetur quo capitaneo nostri de huiusmodi mercationibus quas discaricabuntur in Malicha in reditu exigere debeant dectam mediam pro centinario". ASV, SM, XLV, fol. $1 \mathrm{r}$.

${ }^{25} \mathrm{ASV}, \mathrm{SM}, \mathrm{XLV}$, fol. 9 r.-v.
} 
3. Se pedirá permiso expreso para poder disponer de vino y alimentos exentos de tasas.

4. Se pedirá permiso para que los mercaderes y ciudadanos venecianos puedan cargar y descargar sus mercancías en el puerto de Málaga, o en cualquier otro puerto nazarí, sin coste alguno, quedando sujetas a imposición exclusivamente las mercancías que sean finalmente vendidas ${ }^{26}$.

5. Los nazaríes deberán garantizar libertad y seguridad de movimiento de mercaderes y mercancías por todo el reino en sus desplazamientos por tierra y mar.

6. Se obtendrá la garantía de que el gobierno nazarí no ejercerá represalias sobre la comunidad veneciana ante delitos o faltas cometidos por compatriotas.

7. Se dará libertad a los mercaderes venecianos para que puedan vender sus artículos en la tierra y a mercaderes musulmanes sin restricción alguna.

8. Ante eventuales naufragios en mares granadinos, la mercancía recuperada será devuelta a representantes venecianos acreditados sin coste alguno.

9. Los negocios entre un mercader y ciudadano veneciano y otro de cualquier origen y nacionalidad, cristianos o musulmanes, serán supervisados por el cónsul véneto.

10. Se tiene conocimiento del desigual trato dado hasta el momento a los mercaderes venecianos frente a la comunidad de negocios genovesa activa en el reino ("sumus informati quod in dectis locis et partibus mercantur et conversantur multi januenses cum magna prerogativa et inmunitate"). Por ello se encarga al legado que obtenga información detallada acerca de los derechos y las prerrogativas de que disfrutan los genoveses, con el objeto de solicitar igual o mejor trato a las autoridades nazaríes para la futura comunidad veneciana ("comittimus tibi fueris in Malicha ante que vadas ad presentiam decti domini Regis te informare debeas de omnibus daciis quod soluint januenses predecti et habuta plena informatione omnibus procurabis et instabis penes dominum regem predictum quod veneti et cives nostri sint in totum in facto huismodi solutionis et in quibuscumque aliis prerogativus quas haberent ad conditionem januensis predictorum et ad meliorem si meliorem poteris obtinere" $)^{27}$.

11. Los bienes de súbditos venecianos fallecidos en tierras nazaríes deben ser entregados a los representantes consulares o a quien hubiera designado el fallecido para su traslado y entrega a los herederos sin coste alguno.

\footnotetext{
26 "Item cum consuetum sit et sic observatur in Malicha, quod omnis nostri mercatores et cives qui cum galeys nostris vadunt Malicham possant discaricare in Malicha omnis suas mercationes et si illas vendunt soluint drictum suum, et si non vendunt nichil soluint. Et possunt dectas suas mercationes iteraro caricare et sic nobis in quibuscumque aliis locis ac que galie nostre vadunt, observatur quod est justum propterea comittimus tibi procurare debeas quo hoc ponatur in pactis". ASV, SM, XLV, fö. $9 \mathrm{r}$.

${ }^{27} \mathrm{ASV}, \mathrm{SM}, \mathrm{XLV}$, fol. $9 \mathrm{v}$.
} 
12. Se encarga la entrega de regalos al Rey por valor de 200 ducados.

13. Una vez alcanzado un acuerdo satisfactorio para los intereses venecianos, se confía la dirección del consulado al mismo embajador, que ejercerá sus funciones en la sede malagueña. En caso de que no se alcancen unas condiciones suficientes, se verá liberado de dicho encargo, debiendo entonces volver a Venecia.

14. Se aclara que los gastos de la embajada correrán a cargo de las arcas venecianas en caso de éxito de la misión. En caso contrario, dichos gastos correrán a cargo del embajador.

Por fin, de manera inmediata, el 26 de marzo ${ }^{28}$, se provee la cantidad necesaria, 200 ducados, para los gastos de la embajada y se da orden al capitán de las galeras venecianas de la Muda de Flandes para que ponga en marcha el cobro del derecho del $0,5 \%$ sobre las mercancías con que se cubrirán los gastos del consulado.

Todo queda, por tanto, listo y al detalle para el inicio de la misión. Una embajada que, como sabemos por testimonio del mismo Contarini, llega a Granada poco después ${ }^{29}$, siendo recibida con enorme expectación por las autoridades granadinas, que, a juicio del veneciano, se muestran casi ansiosas:

Upon my arrival at Granada, I was received by the Minister of Foreing Affairs, who informed me that his Majesty was extremely anxious to see me. I excused myself at first, on the plea that the distance to the royal residence was fatiguing after my long journey, and that my vestments were soiled and dusty from travelling. But the minister was importunate, representing that it was an extraordinary honour wich his master conferred upon me, since other envoys were usually detained for some time before an audience was granted to them. Hereupon I yielded, retired to my apartments, opened my valise, attired myself in a bright court-suit and prepared to wait upon the monarch. My reception was peculiarly gracious and amiable, and I procured from the King whom I found seated on a thronal dais and sorrounded by his councillors, a charter written in Arabic characters upon red papyrus by virtue of which the subjects of the Republic are placed in the enjoyment of personal security and many special and valuable privileges throughout Granada ${ }^{30}$.

La respuesta desde Granada revela una buena disposición a facilitar el camino a cualquier nueva propuesta que repercuta en el crecimiento comercial de esta tierra. De hecho muestra su disponibilidad para favorecer y facilitar la estancia y negocios de los mercaderes venecianos, y da los primeros pasos para abrir una alhóndiga exclusiva para los mercaderes de esta nacionalidad y para sus agregados en Málaga.

\footnotetext{
${ }^{28} \mathrm{ASV}, \mathrm{SM}, \mathrm{XLV}$, fol. $7 \mathrm{r}$.

${ }^{29}$ Dudamos de la fecha propuesta de 6 de octubre de 1400 , sobre todo teniendo en cuenta la fecha de la firma del tratado definitivo, datado el 23 de mayo del mismo año.

${ }^{30}$ Col. of State Papers (Venice), 1864, I, 53. Recogido de W. CAREW HAZLITT, The venetian republic. Its rise, its growth and its fall, A.d 409-1797, London 1915, vol. II (1457-1797), p. 664. Desgraciadamente no hemos sido capaces de localizar por el momento el testimonio original, por lo que nos yemos obligados a reproducir el extracto traduçido del mismo. Nos parece en todo caso un testimonio valioso y muy ilustrativo de cuanto venimos proponiendo.
} 
Las conversaciones deben de ser tan breves como fructíferas, a juzgar por la fecha de firma del tratado definitivo del acuerdo, sellado en la misma Alhambra en mayo del mismo año, 28 ramadan de $802^{31}$. El evidente interés de las autoridades venecianas, mostrado en la elasticidad de las recomendaciones realizadas al embajador, es secundado por un interés paralelo mostrado por el rey nazarí, patente en la recepción dispensada a Contarini y, sobre todo, en su disposición a satisfacer los deseos de Venecia en los puntos esenciales de sus demandas. Éstas, según se deduce de la lectura de ese tratado, recogen de manera satisfactoria sus principales aspiraciones en materia fiscal y de equiparación de trato respecto a otras comunidades mercantiles. Los principales puntos del acuerdo se resumen de la siguiente manera: mercaderes.

1. Se acuerda la libertad de comercio y movimiento por el reino a sus

2. Se da permiso para levantar una alhóndiga para los venecinanos y para sus agregados en Málaga, junto a las alhóndigas de los demás cristianos.

3. El cónsul recibirá de las autoridades nazaríes 200 doblas de oro anuales y podrá pagar los derechos impuestos sobre las mercancías en el funduk, tal y como hacen los genoveses.

4. Se permite la entrada de avituallamientos para la alhóndiga, tanto de comida como de bebida, sin pagar derechos, igualando así las condiciones de que disfrutan los genoveses.

5. No se contempla la responsabilidad colectiva.

6 . Ante eventuales problemas de venecianos con los indígenas, sólo podrán intervenir en primera instancia las autoridades locales, los alcaides de la fortaleza y de la aduana. Y sólo el cónsul veneciano podrá actuar de juez en la resolución de conflictos.

7. Se garantiza la seguridad en los puertos nazaríes para las naves venecianas $\mathrm{y}$, ante posibles naufragios, se declara que los náufragos no serán retenidos, sus mercancías y bienes no les serán arrebatados. Se comprometen asimismo a ayudar en la reparación de la nave, ofreciendo la posibilidad a patrón y capitán de comprar los materiales necesarios para dicha reparación.

8. No se confiscarán los bienes de quienes mueran en el reino, siendo, por el contrario, entregados al cónsul o a su representante.

9. Se equiparará en materia impositiva la situación veneciana y genovesa, incluyéndose el pago del derecho de trujamanía, y quedando exentos de los derechos sobre oro, plata, perlas y joyas.

10. No se pagarán derechos en el puerto antes de vender las mercancías. Y se permite la permanencia de mercancías en puerto sin pagar nada en un plazo máximo de diez meses. Hasta esa fecha pueden ser recuperadas sin pago alguno de tasas.

\footnotetext{
${ }^{31}$ Archivio di Stato di Genova, Secreta, Serie Diverse, Commemoriali, reg 9, cc. 111-112. Presentado en Apéndice documental 2. Hasta este momento sólo disponíamos de un resumen de dicho tratado extratado por N. JORGA ${ }_{3}$ Notes et extraits pour servir a l'histoire des croisades au XVe siècle. "Revue de I'Orient Latin", 4 (1896) pp. 25-503, espec. pp. 229-230
} 
Con todo ello se atiende a la apertura de un nuevo espacio de comercio para Venecia, conocido y practicado ya con anterioridad y que en este momento se revela lo suficientemente prometedor como para que el gobierno de la Serenísima asuma su organización de manera oficial. En realidad, lo que percibimos a través de este acto es la declaración de una realidad de facto, vigente desde hace ya un tiempo, que se pretende regular: el puerto malagueño es escala de las galeras venecianas que viajan a Flandes tanto en la ida como en la vuelta. Es una escala rentable y en la que las posibilidades de negocios han pasado a ser ya hechos cumplidos desde hace algún tiempo. De hecho, en la misma justificación elaborada por el senado para amparar esta iniciativa declaraban que "est manifestum galee nostre viagii Flandrie singulo anno eundo et redeundo continue vadunt ad locum Maliche, in quo multe mercationes, civum et fidelium nostrorum expeduntur, et pro ut habetur rex Granate, cuius est dictus locus, est bene dispostus ad comoda et fauores..." ${ }^{-32}$. Una vez más las decisiones o iniciativas políticas asumidas por los estados, incluso en el caso de gobiernos tan atentos a su proyección comercial, va a la zaga de la realidad e iniciativa de las gentes de negocios, que están explorando el mercado desde hace tiempo. En los años inmediatamente anteriores al establecimiento del acuerdo, se tiene perfecta consciencia de los intereses existentes en tierras nazaríes para los negocios venecianos $^{33}$ y se identifica con certeza el artículo objeto de su interés, el azúcar, y los puntos de aprovisionamiento a los que deben de acudir. Málaga y sobre todo Almuñécar son los puntos a los que llegarán las naves venecianas, gracias a salvoconductos dispensados por el rey granadino, a recoger la mercancía. Y dado el alto interés que presenta su aprovisionamiento ${ }^{34}$, conviene asegurarse el apoyo de las autoridades nazaríes en cada viaje de la muda, una recomendación expresada de forma repetida en los incanti de las mude desde $1395^{35}$, que, desde luego por lo que sabemos acerca de los trayectos cumplidos por las mismas, efectivamente están tocando el puerto

${ }^{32} \mathrm{ASV}, \mathrm{SM}$, reg. XLV, fol. $3 \mathrm{r}$.

${ }^{33}$ En 1400 , por ejemplo, un tal Simone Bonafè declara que "va a stare a Sobilia e uno suo fratelo a Malicha", siendo presentados por la sociedad florentina de los Sernigi en Venecia a la compañía Datini de Barcelona. Archivio di Stato di Prato, Datini 920, Carteggio Valenza-Bar cellona, 30.4.1400.

${ }^{34}$ Doris STÖCKLY, Commerce et rivalité a Chypre. Le transport du sucre par les Vénitiens dans les années 1440, d'après quelques documents génois, en Oriente e Occidente tra Medioevo ed Età Moderna. Studi in onore di Geo Pistarino (L. BALLETTO a cura di), Genova 1997, pp. 1133-1144; E. CANAL, F. COZZA, L. LAZZARINI, G. VITTA LAZZARINI, La lavorazione dello zucchero a Venezia documentata dal ritrovamento di forme e cantarelli nella laguna veneta. "Padusa. Bollettino del Centro Polesano di Studi Storici Archeologici et Etnografici", XII (1976), pp. $125-142$.

35 “In super quo bonum est captare benivolentiam cum domino Maliche ordinetur quo capitaneo dictarum galearum possit si capitaneo videbitur ire cum dectis galeis ad dectum locum Maliche vel de Melecha et si illuc accedet presentare debeat (exenia) ad valorem ducatorum Lta de bonis galearum in illis rebus quos dectis capitanei et patronis videbuntur non possendo stare in decto loco ultra dies tres conputatis diebus accesos et recessus procurando factis (exeniis) predectis habere salvos conductus ab eodem dominoo pro galeis, navigis et mercationibus nostris ad partes sibi subiectas cum suis mercationibus declarantibus pro quo maiori tempore poterit obtinere". ASV, SM, XLIII (1395), fols. 190 r. -192 v. 1395,8 de enero. En parecidos términos se expresan las regulaciones de los incanti de las galeras de Flandes de los anos sucesivos hasta 1400. ASV, SM, XLIV (1395), fols. 29v. -31v.; 76 r.-78 r.; 135 v.-138 v. 
malagueño ya en estos años finales del XIV ${ }^{36}$. Es, por tanto, una realidad de facto vigente desde hace ya algún tiempo, tal y como ellos mismos declaran, y llena de expectativas de progreso y rentabilidad futura y bastante inmediata. Hasta tal punto es así que los cálculos del gobierno veneciano en materia de financiación del próximo establecimiento consular, para el que se instituye el cobro de un derecho sobre las operaciones mercantiles vinculadas a esta tierra granadina, calculan no sólo cubrir las necesidades previstas con lo recaudado, sino incluso superarlas (quod superhabundavit ponat ad rationem nostri comunis).

\section{LOS INTERESES A TUTELAR}

Evidentemente la creación de una colonia mercantil estable en uno o varios mercados del territorio es fundamental. Estamos con toda certeza, a pesar de los escasos datos de que disponemos hasta el momento, y guardando las debidas distancias, frente a uno de los muchos establecimientos comerciales que tutela Venecia en el Mediterráneo, posiblemente similar a los abiertos en áreas de presencia veneciana mucho más consolidada, donde se ve con mayor claridad la existencia de un sistema de asentamientos jerarquizados ${ }^{37}$.

En tierras nazaríes Venecia asume una responsabilidad organizativa y de control de sus mercaderes, sean quienes sean y segura, en todo caso, que son una comunidad con la fortaleza suficiente como para que la República reconozca su labor, y se decida a regular y a regularizar definitivamente las relaciones entre los dos estados. El interés veneciano por proteger a sus súbditos e intereses de los mismos en Granada se plasma de manera más que evidente en la creación de una figura consular, origen de una nación oficialmente constituida $^{38}$. Es un cargo dependiente directamente del gobierno veneciano, que lo sostiene y mantiene, con todo el equipo de colaboradores, y que muestra sus expectativas en la asignación de un salario elevado (400 ducados anuales). Para ello se acude a fórmulas de financiación gestionadas desde el gobierno y que contemplan el cobro de un derecho del 0,5 por ciento sobre las mercancías y mercaderes operantes en tierras granadinas destinado exclusivamente a cubrir los gastos de esta representación oficial veneciana en Málaga (XLV, fol. 7 r. Comisión a galeras de Flandes de 1400 así lo contempla).

El delegado veneciano mantiene una sede que debe de tener un referente topográfico definido, una alhóndiga, cuya ubicación exacta en

\footnotetext{
${ }^{36}$ ASV, D, Filza 1171. Quaderno di Carichi. 1394. Cargamento de las 4 galeras venecianas con destino a Flandes y parada en Málaga para descargar pimienta, paños, tafetán y papel; Idem. filza 1171, Carichi di navi, Doc. 51, 1396. Cargamento de las 4 galeras venecianas con destino a Flandes y parada en Málaga para descargar plomo, clavo, pimınta y paños.

${ }^{37}$ Bernard DOUMERC , La Tana au XVe siècle: comptoir ou colonie?, en Etat et colonisation au Moyen Age et à la Renaissance (M. BALARD dir.), Lyon, 1989, pp. 251-266.

${ }^{38}$ Giovanna PeTti BALBI, Negoziare fuori Patria. Nazioni e genovesi in età medievale, Bologna, 2005.
} 
Málaga nos es desconocida por el momento. Pero existe en algún momento, y además se consigue el compromiso del estado nazarí de mantenerla integramente. En realidad los venecianos se ciñen a modelos ya puestos en práctica por ellos mismos y por tantos otros mercaderes italianos en otras tierras musulmanas ${ }^{39}$. La práctica, por ejemplo, de cargar los costes de mantenimiento de la alhóndiga a las autoridades receptoras resulta ser bastante común en el mundo árabe, así como la aportación pecuniaria que deben realizar para el mantenimiento del consulado, que en esta ocasión asciende a la cantidad de 200 doblas anuales ${ }^{40}$. Pero evidentemente lo que centra el interés de los venecianos es conseguir unas condiciones fiscales favorables y que los equipare, al menos, a la situación que disfruta la nación genovesa. Deben pagar derechos sobre mercancías exclusivamente que vendan o compren allí, no sobre las que descarguen, que pueden recuperar si no se han vendido, sin coste alguno. Asimismo se pedirán los mismos beneficios que se sabe que tienen los genoveses, que disfrutan de una situación ventajosa fiscalmente respecto al resto de comunidades (cum magna prerrogativa et inmunitate). Algo que a estas alturas se considera ya posible. De hecho, poco después sabemos de otra comunidad mercantil de influencia ascendente en la región, la catalana, que consigue acogerse a esta situación de igualdad de trato fiscal $^{41}$.

Porque, ¿para quienes trabaja esa institución? ¿qué intereses tutela?

De la lectura y condiciones del tratado se deduce la existencia y actividad de un grupo de mercaderes venecianos, residentes de manera más o menos estable, al menos en la ciudad de Málaga, sede del consulado. Ahora bien, identificar a sus miembros es tarea mucho más difícil. Primero precisamente porque no sabemos hasta qué punto se trata de miembros estables, salvando por supuesto a los representantes de la delegación oficial.

La verdad es que para éste nuestro caso poco, apenas nada, podemos decir acerca de la comunidad de negocios veneciana que se pudo constituir en el reino, o que estaba ya activa antes de su oficialización. Porque, aparte de la identidad de los dos encargados consulares, y de alguna brevísima noticia anterior, no conocemos nada acerca de la categoría, entidad o identidad de los miembros de una comunidad que sin embargo existió sin duda alguna.

¿Qué podemos decir de este Bernardo Contarino que encabeza la operación? Poco, en realidad. Miembro de una de las familias venecianas más implicadas en el comercio de Estado desarrollado a través del sistema de

\footnotetext{
${ }^{39} \mathrm{~F}$. GIRARDI, Venezia e il regno di Tunisi.... Una breve y estupenda descripción del modus operandi generalmente utilizado por los mercaderes italianos de la época en tierras islámicas, concretamente en el norte de Africa en G. PETTI BALBI, Las ciudades maritimas italianas y el Norte de Africa.

${ }^{40}$ David ABULAFIA, Las redes consulares del Mediterráneo: función, orígenes y desarrollo, en Mediterraneum. El esplendor del Mediterráneo medieval. Siglos XIII-XVV, Barcelona 2004, pp
339-351.

${ }^{41}$ R. SALICRÚ, La Corona de Aragón y Génova.
} 
mude $e^{42}$, Bernardo es un mercader con experiencia en el mundo de los negocios con países islámicos. De hecho lo encontramos pocos años antes, en 1394, actuando al más alto nivel en Beirut con ventas al mismo sultán mameluco ${ }^{43}$. Su edad ya avanzada (el uechio e nobele e lial de tute cortexie miser Bernardo Contarini...) y su buen conocimiento del árabe, que lo lleva a actuar él mismo como intérprete en su embajada primera con el rey (uostro mesazier Bernardo Contarini siando turzimano veriteuele entro un el uostro messazier... ) así nos lo muestran. Pero desde luego queda claro el interés de la familia en establecerse en esta tierra, con la que mantiene negocios más adelante ${ }^{44}$.

Respecto a Andrea Corner, sabemos del rango de la familia, perteneciente también a la nobleza veneciana y también de las más implicadas en la organización y gestión del sistema de convoyes estatales. Y cómo no, recordamos la implicación de esta familia en los negocios azucareros venecianos desarrollados en Chipre a partir de iniciativas privadas y que tienen ya una larga trayectoria. Pero por supuesto no estamos ni mucho menos en condiciones de afirmar que cierto interés por esta nueva región azucarera en pleno auge motive la presencia, breve en todo caso, de un miembro de esta familia a la cabeza del consulado malagueño.

De manera que lo único que podemos avanzar a partir de ahora no dejan de ser generalidades por el momento que, sin embargo, dejan la puerta abierta a estudios más pormenorizados que deberían realizarse en este campo.

Si podemos decir, por ejemplo, que en general debe de tratarse de estancias de carácter temporal, una temporalidad más breve que amplia. Una serie de prácticas, comunes en otros consulados de la región, si, aunque no por ello dejarían de indicar, a nuestro entender, la naturaleza de unas estancias poco estables y generalmente no arropadas por el núcleo familiar, en la mayoría de los casos. Así, cuando se incluye entre las competencias judiciales que el cónsul ostenta entre la comunidad mercantil veneciana en suelo nazarí, la obligación de gestionar bienes y mercancías de miembros de la comunidad que mueran en suelo granadino, y se plantea como una cláusula importante de respeto de las autoridades granadinas hacia las competencias internas del representante veneciano, entendemos que se trata de una cuestión de importancia en una comunidad que practica esta "temporalidad". O cuando se incluye como un elemento clave del equipo permanente de asistentes del

\footnotetext{
${ }^{42}$ Stockly sitúa a la familia Contarini a la cabeza de los clanes familiares implicados en este negocio, a tenor del número de patrones y capitanes pertenecientes a la familia. Ello, sin embargo, no implica a su juicio una hegemonía y ni mucho menos monopolio de estas familias en el sistema de incanti estatal, sino simplemente un predominio político de estos clanes en el gobierno veneciano. En todo caso sí que apunta claramente la dirección de los negocios que nutren a estas familias y, obviamente, sus intereses económicos. D. STÖCKLY, Le système de l'Incanto des galées du marché ..., pp. 304 y ss.

${ }^{43}$ ASV, SM, XLIII (1394), fol. 10 r. 1394, 4 de junio. En estas fechas aparece Bernardo Contarino, posiblemente el mismo que después será cónsul en Málaga, activo como mercader en Beirut, donde reclama el cobro íntegro por parte del sultán de Alejandría, de una cantidad de cobre que le ha vendido junto con otros mercaderes y que no ha cobrado.

${ }^{44}$ José Enrique LóPEZ DE COCA, Comercio exterior del reino de Granada, en El reino de Granada en la época de los Reyes Católicos. Repoblación, comercio, frontera II, Granada 1989 , pp. $129-180$, espec. p. 147.
} 
cónsul la figura de un trujamán, se reconoce su función imprescindible, a pesar de los riesgos que pueden implicar el cierre de negocios o tratos delicados con el concurso de un intermediario.

Y es que evidentemente no deben de permanecer el tiempo suficiente en la mayoría de las ocasiones como para aprender la lengua local ${ }^{45}$, empaparse de las costumbres de la población indígena y ni mucho menos renunciar a las propias, que deben de ser preservadas inexcusablemente. En este sentido la petición expresa de permiso para poder consumir vino dentro de la alhóndiga y la inclusión, posiblemente, de un cura en el equipo del cónsul, tal y como se aludía en el primer documento, trasciende la pura anécdota. En realidad se trata de una petición ya conocida, e incluso común, en otros tratos con territorios islámicos y denota efectivamente un modo de hacer particular, marcado por una voluntad abierta de preservación de la propia identidad, que caracteriza las relaciones de estas comunidades de negocios con las plazas de la órbita islámica ${ }^{46}$.

Pero esta temporalidad no resta valor a la actividad de una comunidad que se muestra extraordinariamente dinámica. De hecho otro de los rasgos que podemos apuntar sólo a nivel de generalidad, es su carácter abierto e interactivo con otras comunidades del área. Algo, por otro lado, absolutamente lógico en un mundo como éste de los negocios, en el que buena parte del éxito comercial de las empresas depende de la calidad de los conocimientos exhaustivos de los mercados que participan en la operación y en la capacidad misma de conectar y cruzar intereses y situaciones convergentes. A lo largo de la exposición han ido surgiendo pequeños destellos del buen funcionamiento de esta red de contactos. Una trama que inicia su marcha desde el momento mismo del establecimiento de intereses en la región por parte de iniciativas particulares y que es asumida de manera natural como parte de la política de protección de intereses comerciales por parte del gobierno veneciano. Recordemos el destino de miembros de la misma familia a plazas vecinas como Sevilla y Valencia al filo del $400^{47}$. Y cómo las mismas gestiones del consulado veneciano en Sevilla incluyen en todo momento los servicios de un trujamán que agilice eventuales contactos con espacios vecinos ${ }^{48}$. Y los contactos son estrechos también en la otra orilla del Estrecho, mostrándosenos fuertes relaciones al más alto nivel con la comunidad de negocios veneciana asentada en Túnez, con implicación directa en los contactos tanto por parte del

\footnotetext{
${ }^{45}$ Antonio Peláez Rovira, Sobre el uso de la lengua árabe en el comercio genovés con el Islam occidental bajomedieval, en Atti della Società Ligure di Storia Patria, XLVII (2007), pp. 143-176, espec. pp. 162 y ss. Roser SALICRÚ, ¿Ecos de aculturación? Genoveses en el mundo islámico occidental, en Genova una "porta" del Mediterraneo (L. GALLINARI a cura di), Genova 2005, vol. I, pp. 175-196.

${ }^{46}$ Adela FÁBREGAS, Comunidades mercantiles extranjeras en el reino nazarí de Granada: el mundo de los negocios como espacio de interculturalidad, (en prensa).

${ }^{47}$ ASP, D 920, Valencia-Barcelona, 30.4.1400. Véase nota 39.

${ }^{48}$ En 1402 la elección de un cónsul para Sevilla con salario de 400 ducados anuales contempla también los gastos de personal de otrọ tipo, entre el que se debe de contar un trujamán que sepa árabe y latín ("quorum unius sit trucimanus et sciat ydioma arabum et latinum") y un notario. ASV, SM, XLVI (1402), fol. 8 r.
} 
cónsul granadino (... mi mancha alguna letera da Voxia yo dovea aver. Per ciò vi prego fate veder se tra queli merchadanty de lly le ffosse fatica in averlla e mandatemelle per luy presto pode. $)^{49}$, como del tunecino ${ }^{50}$.

\section{EPÍLOGO}

De todos modos, ni por relevancia numérica, ni por duración de la experiencia y ni mucho menos por capacidad para influir de ningún modo en la dinámica interna del reino por encima del desarrollo de sus intereses estrictamente comerciales, se puede hablar de una experiencia que vaya más allá.

Y es que las expectativas iniciales de ambos estados no parecen verse cumplidas de manera totalmente satisfactoria, según se desprende de la lectura de las actas posteriores del Senado. De hecho, los problemas de financiación de este consulado son muy rápidos y desde poquísimos años después, el mantenimiento del mismo parece responder exclusivamente a intereses diplomáticos más que estrictamente económicos. El siguiente cónsul veneciano, Andrea Corner, activo ya en el cargo desde 1402, está llamado a cerrar la representación consular en Málaga a instancias del Senado, que ha llegado ya a la conclusión de que "habere consulem in Malicha non redundant ad illud proficuum quo credebatur pro factis nostris" 51 . Sólo la evidente amenaza de perjudicar los intereses comerciales venecianos aún activos en la zona, y, por qué no, instado por una cierta presión por parte de las autoridades nazaríes (presión que podría dejar entrever cierto tono amedrentado de la rectificación emitida por la autoridad veneciana pocos días después, el 13 de abril de 1402), lleva a Andrea Corner a reconsiderar la oferta de permanencia en el puesto a cambio de un sueldo de 300 ducados anuales $^{52}$. Una oferta aceptada por su parte, ya que sabemos de su actividad consular a lo largo del año de 1403 por otras fuentes ${ }^{53}$, aunque con posterioridad decide renunciar al cargo antes de consumir el plazo de tiempo de tres años asignado a la dignidad consular. De hecho justo dos años después, en abril de 1404, Corner comunica su intención de abandonar la plaza. Su decisión, cuyos motivos se nos escapan, abre una crisis en la que subyacen problemas económicos serios para el mantenimiento de la institución, que resulta cara al gobierno veneciano pero que de ningún modo tiene intención de cerrar para no soliviantar al rey nazarí. Por ello, a pesar de reducir el estipendio del representante consular hasta el punto de que nadie quiere ostentar el cargo por los 200 ducados

\footnotetext{
${ }^{49}$ ASP, D, Málaga-Valencia, 14.6.1403. El destino de la carta revela también contactos con el mercado valenciano.

${ }^{50}$ Vid infra nota 57.

${ }^{51}$ ASV, SM, XLVI (1402), fol. 11 r. 1402, 28 de marzo

${ }^{52} \mathrm{ASV}, \mathrm{SM}, \mathrm{XLVI}(1402)$, fol. 14 vto. 1402,13 de abril

${ }^{53}$ ASP, D, 999 (Carteggio comune di Malaga).1403, junio, 14. Málaga.
} 
anuales ofrecidos ${ }^{54}, 250$ más tarde, no dudan acerca de la importancia estratégica del mismo, que debe mantenerse abierto a toda costa, recurriendo, incluso, a la comunidad veneciana de Brujas y a su mismo representante ${ }^{55}$. Asî es como a duras penas logran mantener la institución abierta, aunque la prioridad en estos momentos, más que buscar un digno representante para la misma, parece ser la de contener los costes de esta representación al máximo, de manera que, cuando se procede a la sustitución del cónsul, cuya identidad ya no conocemos, en 1406, se aclara que capitaneo nostro galearum Flandrie quo debeat providere inde de eligendo ibi unium consulem sue novilem sive popularem quo minori expensa et onere poterit et cum illis modis et conditionibus que sibi videbuntur ${ }^{56}$.

Es la última noticia de que disponemos acerca de la aventura consular veneciana en Granada. Pero evidentemente su declive no arrastra los intereses comerciales ya establecidos en la zona, que mantienen su vigencia, tal y como hemos podido entrever gracias a la continuidad de las escalas nazaríes de las grandes rutas de las mudas occidentales. Es más, las relaciones, las buenas relaciones e intereses comerciales en Granada y con los cuadros de poder nazaríes se reflejan de manera evidente en episodios como el trasladado en 1421 por el cónsul veneciano en Túnez. Un embajador nazarí es portador de cantidades de dinero propiedad de mercaderes venecianos residentes en Túnez y que deben de tener intereses comerciales en tierras granadinas. El dinero, entregado al sultán Abu Faris, no llegará a sus propietarios, siendo retenido por el sultán como garantía de cobro de una deuda pendiente, lo que suscita las protestas de mercaderes y del gobierno de la Serenísima ${ }^{57}$. Desde luego, a pesar de las dificultades por las que parece atravesar el mantenimiento del consulado en tierras nazaríes a principios de siglo, queda claro que los intereses comerciales son aún relevantes y suficientes para preservar estancias, negocios y escalas en la zona, aún cuando no disponemos por el momento de información más concreta sobre la cuestión. Unos contactos que deben de ser aún prósperos incluso en fechas avanzadas. Sabemos todavía de la llegada de las galeras de Flandes a Málaga durante los años 30 del siglo $^{58}$. Pero sobre todo recordamos que nuevas mudas establecidas con posterioridad, ya entrado el siglo XV, como la Muda de Berbería, inaugurada en 1437, contemplan también las escalas nazaríes de manera creciente, tanto en relación a los puertos tocados, Málaga y Almería desde 1454, como respecto a las jornadas

${ }^{54}$ ASV, SM, XLVI (1404), fol. 127 v. 1404, 29 de abril.

${ }^{55}$ ASV, SM, XLVI (1404), fol. 147 vto. 1404, 9 de agosto.

${ }^{56}$ ASV, SM, XLVII (1406), fol. 63 r. 1406, 29 de julio.

${ }^{57}$ ASV, SM, LIII, octubre de 1421. Información recogida en Alberto SACERDOTI, Venezia e il regno hafsida di Tunisi. Trattati e relazioni diplomatiche (1231-1534). "Studi Veneziani" VIIII
(1966), pp. 303-346, espec. pp. 332-333. Agradezco al Dr. Gónzález Arévalo la referencia bibliografica.

${ }^{58}$ ASV, SM, LIX fols. 29 r.-32 r (encanto de galeras de Flandes del año 1433); ASV, SM, LIX fols. $85 \mathrm{v}$.- $86 \mathrm{r}$. (encanto de galeras de Flandes del año 1434); ASV, SM, LIX fols. 142r.-v (encanto de galeras de Flandes del año 1435); ASV, SM, LX, fols. 60 vto-63 r. (encanto de galeras de Flandes del año 1437); ASV, SM, LX fol. 128r. -130 vto (encanto de galeras de Flandes del año 1439). 
de parada contempladas para estas escalas, dos o más, en detrimento de las escalas castellanas de las costas meridionales, Cádiz y Sanlucar de Barrameda $^{59}$.

Los barcos venecianos siguen llegando a Málaga, incluso en fechas muy tardías de este mismo siglo (en los años 70 aún hay testimonios del acceso de las galeras venecianas a Málaga). Pero en todo caso ésta ya no es una escala oficial.

${ }^{59} \mathrm{D}$. STÖCKLY, Le système de l'Incanto des galées , pp. 169 y ss. La llegada de la muda de Berbería a las costas del reino de Granada ha sido tratada por José Enrique LÓPEZ DE COCA, Granada, el Magreb y las galeras mercantiles de Venecia en tiempo de los Reyes Católicos y Carlos I», en 1490 en el umbral de la Modernidad. El Mediterráneo europeo y las ciudades en el tránsito de los siglos XV-XVI (J. HINOJOSA MONTALVO y J. PRADELLS NADAL eds.), Valencia 1994, vol. I, pp. 23-55, quien aclara la preferencia veneciana por el puerto almeriense. 


\section{APÉNDICE DOCUMENTAL}

[sin fecha]

Copia de la carta del rey de Granada, Muhammad VII, al Dogo de Venecia, Antonio Venier, dando cuenta de su encuentro con Bernardo Contarino, embajador de la Serenísima.

Archivio di Stato di Venezia, Secreta, Serie Diverse, Commemoriali, reg. 9, fol. 111 r (114), n. 205.

Copia Litere Serenissimi Regie Granate scripte in arabico in papiro rubeo translate de arabico in latinum.

In nome de Dio sia de lo Re de i mori seruo de Dio e quelo che crede in Dio Machomet fio del Signor di mori fio de Joseph fio de lo Re de i mori fio del Re Abdelo fio del Re de i mori Joseph fio del Re de i mori Ysmayl fio de Naser Dio lo defenda, scriue a lo Doxe de Veniesia che e forte caualier e rico e de gran sangue grando como cristiano che sia al mondo homo de veritade in dicti e fati. El nobele Antonio Venier, el comun de Veniesia e i çitilomeni che xe in Venecia Dio i guarda e defenda tuti. Al doxe e a la comunita saludi assai. Iu ue scriuemo de lanbra alta de Granata Dio la defenda e guarda da tuti pericoli gratia sis dada a Dio e dia gratia a un altri. Io me tegno contento de vu altri e per questo gratia al ducha gran signor e al nobele comun de Veniexia che le vegnudo a un el uostro misaçier el uechio e nobele e lial de tute cortexie miser Bernardo Contarini dio lo defenda, de la carta de la uerita che el doxe el comu de Veniexia a dado che se diebia presentar mandassemo che vignisse in le nostre man el de a dito tute cosse che vu mandasse a dir e tute cosse che vu aue ordenado e no a manchado niente. El ne a dito de la amistade che vu aue con un e io el credo de la uostra cognosança e amistade un semo contenti de zo che vu dixe e nu Re me tegno contento de la uostra amistade e si o fato tuto quelo che vu ave domandado per el uostro ambasador de tute cosse che la dito dauanti da un. Io ue digo a vu altri che me mande a dir de tute cosse che vu aue besogno in tuto el mio regno e tute cosse che vu ave de mestier per uostro amor io el fare che Dio ue lassa ayer ben. El re Saluda al doxe el comun cum tuti i zintilomeni. Fata di XXVIII de la nostra quarexema in Granata.

Tergo. Al doxe de Veniexia grando e ebon Antonio Venier e al comun de Veniexia e ai grande e a i boni zentilomeni che Dio sia sanita e alegreza a tuti e Dio del cielo sia cum elli. 
1400, mayo, 23. (Ramadán 802).

Copia del acuerdo de comercio entre el reino de Granada y la República de Venecia

Archivio di Stato di Venezia, Secreta, Serie Diverse, Commemoriali, reg. 9, fol. 111 v (114), n. 206.

Copia pactorum Inter Serenissimum Regem Granate et Comune Venetiarum translatorum de arabico in latinum.

In nome de Dio sia. Sapia zascum che vedera questa carta e chi la vedera e oldira che Un Re de Granata seruo de Dio che crede in Dio el re Machomet fio del nostro Signor Joseph fio del re Machomet fio del re signor Josef e fio del Re Ismayl fio de Naser Re de Granata e de Malicha e de Ronda e de Zubeltar e de Almeria e de Vera e de Basta e de Godie, Re de Granata e Re de i mori chel vene a un el mesazier del doxe de Veniexia gran signor nobele e ben vouido da tuta la zente del mondo e quelo che tuto el mondo parla bem per elo miser Antuonio Venier doxe de Veniexia e uardaor de tuta soa zente el mesazier del comun de Veniexia Bernardo Contarini do a portado carta del doxe e del comun de Veniexia scrita in latin con el so segno su la sua letera el fo dauanti da mi per le parole chel ne uoleua dir per parte uostra e credessemo a la parola del uostro mesazier Bernardo Contarini el domanda da un la paxe e la amistade entro vu e un e fico elo la paxe bona e ferma per sempre e cossa ueriteuele che no i se puo mudar e uegna seguir tuti i veneziani in nostra terra e in tuto el nostro regno per mar e per terra e con le persone e con lauer e con tute mercadantie e con tuti i uostri nauili e con tuti compagni vignera cum vostri nauili de uostra terra e de terra stranziera che sia suxo i uostri nauili de tute cosse che entro vu e un el uostro mesazier ne a dito tute cosse che vu doande e domanda nostra cognosanza e un credemo le parole che nea a dito e credemo chel dixe veritade e auemo fato con esso la paxe e con $\mathrm{Vu}$ ducha e con el comun de Veniexia e con i zintilomeni e con tuta uostra zente e con tuto zo che sia da uostra parte un lauemo fato per un e per nostra zente e per tuti queli che xe de soto del nostro regno e che non sia algun che ossa dir contra un altri e comandassemo chel se fesse questa carta e nu receuemo le cosse vostre soura de un como receue Bernardo Contarini uostro mesazier le nostre cosse soura de lu. E questo fato xe soura de un Re e de vu altri io lo receuido e fato intro un e vui altri, la prima cosa che vu domande sie che un debiemo far un fontego in Malicha a pruo deli fontegi de li altri cristiani che sia per venetiani solamente che istia e habita seguramente la soto lombra de lo Re e tuti sia seguir de le persone e de lauer e con tuti queli che sera con vu o che se partira da vu tuti sia seguir per la parte del re e deli pati che entro vu e un. E volemo chel consolo che sera qua per vu habia CC doble doro da un per cadauno anno e questo femo per honor e per cortesía. E uolemo che tute cosse che ue besogna per uostro manzar e per uostro ver vu le posse meter intro lo uostro fontego senza pagar alguna cossa como e usanza de i zenoesi. E tuti i mercadante che vegna o uada per lo nostro regno che sia defendudi e guardadi in ogno luego lache i desmonta e no sia nesun che $\mathrm{i}$ ofenda e che i uenda e copra segondo usanza. E sel sera algun di nostri che faza mal in tuto el mio regno che non sia dada pena a altri cha lui solo e che no possa domandar i altri chi a colin che auera fato el mal e che algun non habia mal per lo pecado de altri. E tuti queli che vignera con mercadantia possa vendedr a chi i plaxera senza algun impazo e che i mercadi fati con 
i mori sia conseruadi. Anchora se vegniera alguna deferentia tra moro e venetian che non sia algun che habia libertade soura i veneziani excepto lo archayto del castello e lafizo e lo Archayto de doana. E che tuti uostri nauili sia guardadi e defexi in tuti nostri porti e se algun uostro nauilio naufragasse in porto o fuor de porto che le persone e zo che se recatasse sia salue e segure e che algun no faza forza e tute cosse che fosse in nauilio cussi de mercadantia como de ayer tute cosse che se recatasse sis dado al paron e ai mercadante e che i possa comprare da tuti feramenta, legname, pegola e stopa per reparation de i suo nauilii. E se algun moro ouer altri che se sia auera diferentia con algun venetian chel consolo solo sia zudexe e debia defenir le deferentie como i pareza che sia zusto. E uolemo che se algun mercadante venetian morira in lo nostro regno che i beni del morto prouegna in man del consolo over chi sera per lo consolo e che algun non possa prender de i beni del morto. E uolemo debie pagare el nostro dreto como paga i zenouesi, excepto de oro, arzento, perle e zoye et per cento debie pagar del dreto e la vsanza de la turzimania. E uolemo che sel vi gaera algun nauilio al porto che descarga de le mercadantie chel non sia tignudo a pagar el dreto infino che non auera vendudo la mercadantia, e se el uende chel paga e che i mercadante no sia streti a vender e possa recargar le soe mercadantie infino X mexi e da puo passado el dito termene debia pagar el dreto como// se le cosse fosse vendude e al ducha e al comun dixe che questi e li pati con lo uostro messazier zintilomo Bernardo Contarini e auemo receuudo questo e soura questo fato la paxe e femola con tuti del nostro regno, e soura questo demo nostra veritade e un otigueremo ben e guardaremo la paxe e semo certi de questo e hauemo fato scriuer a questi testemonii in 2 carte scrite in arabesco e in tute do auemo scrito de nostra man e femo testemonianza per un medemi e in cada una de le nostre carte auemo messo la nostra bola e si lauemo fate con testemonianza e scrite con testemonianze del cadi che e fuora tuti i cadi del nostro egno. E vna de le carte saluemo per un e lastra demo al uostro mesazier e per questo sia seguro cadaun uostro che uora vegnir al mio regno. Scrita questa carta del nostro comandamento in Lambra de Granata del tempo che le fata a vltimo di del nostro ramadan in ano 802, segondo usanza di mori e testemoni de un e del uostro mesazier Bernardo Contarini siando turzimano veriteuele entro un el uostro messazier e testimoniando qua Laguzi grando homo papresso el Re. E testemonii se de questo Abenasym e testimonia sora questo el tesorier del re che nome Anbesuba. E testimonia Mahomet Alcaysi casi sora i cadi e lore scriue cum su aman tuto esser vero como e scrito.

Fecha de recepción del artículo: junio de 2010.

Fecha de aceptación y versión final: septiembre de 2010. 